\title{
Comparative Efficacy of Multiple Therapies for the Treatment of Patients With Subthreshold Depression: A Systematic Review and Network Meta-Analysis
}

\begin{abstract}
Xiumin Jiang ${ }^{1}$, Yongxin Luo 2 , Yiwen Chen ${ }^{1}$, Jinglan Yan', Yucen Xia', Lin Yao Xiaotong Wang ${ }^{1}$, Su He ${ }^{1}$, Feixue Wang ${ }^{3}$, Taiyi Wang ${ }^{3}$ and Yongjun Chen ${ }^{1,3,4 *}$

1 South China Research Center for Acupuncture and Moxibustion, Clinical Medical College of Acupuncture, Moxibustion and Rehabilitation, Guangzhou University of Chinese Medicine, Guangzhou, China, ${ }^{2}$ Department of Biostatistics and Preventive Medicine, School of Basic Medical Sciences, Guangzhou University of Chinese Medicine, Guangzhou, China, ${ }^{3}$ Research Institute of Acupuncture and Moxibustion, Shandong University of Traditional Chinese Medicine, Jinan, China, ${ }^{4}$ Guangdong-Hong Kong-Macao Greater Bay Area Center for Brain Science and Brain-Inspired Intelligence, Guangzhou, China
\end{abstract}

OPEN ACCESS

Edited by:

Gang Chen,

Jinan University, China

Reviewed by:

Guo-qing Zheng,

Zhejiang Chinese Medical University,

China

Liu Lan Ying,

Tongde Hospital of Zhejiang Province,

China

*Correspondence:

Yongjun Chen

chyj417@126.com

Specialty section:

This article was submitted to Emotion Regulation and Processing, a section of the journal

Frontiers in Behavioral Neuroscience

Received: 09 August 2021 Accepted: 13 September 2021 Published: 08 October 2021

Citation:

Jiang X, Luo Y, Chen Y, Yan J, Xia Y, Yao L, Wang $X$, He S, Wang F Wang $T$ and Chen $Y$ (2021) Comparative Efficacy of Multiple

Therapies for the Treatment

of Patients With Subthreshold Depression: A Systematic Review and Network Meta-Analysis. Front. Behav. Neurosci. 15:755547. doi: 10.3389/fnbeh.2021.755547
Background: Subthreshold depression (SD) is considered to be the precursor stage of major depression, which is correlated with functional impairment and increased suicide rate. Although there are multiple therapies for the treatment of SD, the comparison and efficacy of various methods has yet to be evaluated. This study aimed to evaluate the efficacy of different therapies by performing a Bayesian network meta-analysis.

Methods: We searched eight databases on April 3, 2021. Center for Epidemiologic Studies Depression Scale (CES-D), Beck Depression Inventory scale (BDI), the Patient Health Questionnaire-9 (PHQ-9), and the Kessler Screening Scale for Psychological Distress (K-6) were used as efficacy outcomes. This Bayesian network meta-analysis used a fixed-effects model.

Findings: Twenty-one randomized controlled trials involving 5,048 participants were included in this study. The results suggested that electroacupuncture (MD -12.00 , 95\% Crl -15.00, -10.00), conventional acupuncture plus wheat-grain moxibustion (MD -9.70, 95\% Crl -14.00, -5.30), and the Chinese traditional peripateticism pill plus group counseling (MD -9.00,95\% Crl -11.00, -6.70) had better efficacy than the control group (CG) in improving CES-D. For BDI outcome, bright light therapy (MD $-9.70,95 \% \mathrm{Crl}-13.00,-6.00)$, behavioral activation program (MD $-5.70,95 \% \mathrm{Crl}$ $-6.10,-5.40$ ), and dim light therapy (MD -6.30,95\% Crl -10.00, -2.20) were better than the CG. Tai chi (MD $-3.00,95 \% \mathrm{Crl}-4.00,-2.00)$ was better than CG for PHQ-9 outcomes. Telephone-based cognitive behavioral treatment (MD $-2.5095 \% \mathrm{Crl}-2.70$, -2.30) was better than the CG for K-6 scores.

Conclusion: Our results suggest that electroacupuncture or bright light therapy appear to be the better choices in the treatment of SD. This study provide new insights into clinical treatment selection and may aid the development of guidelines for the management of SD.

Keywords: subthreshold depression, multiple therapies, network meta-analysis, systematic review, Bayesian analysis 


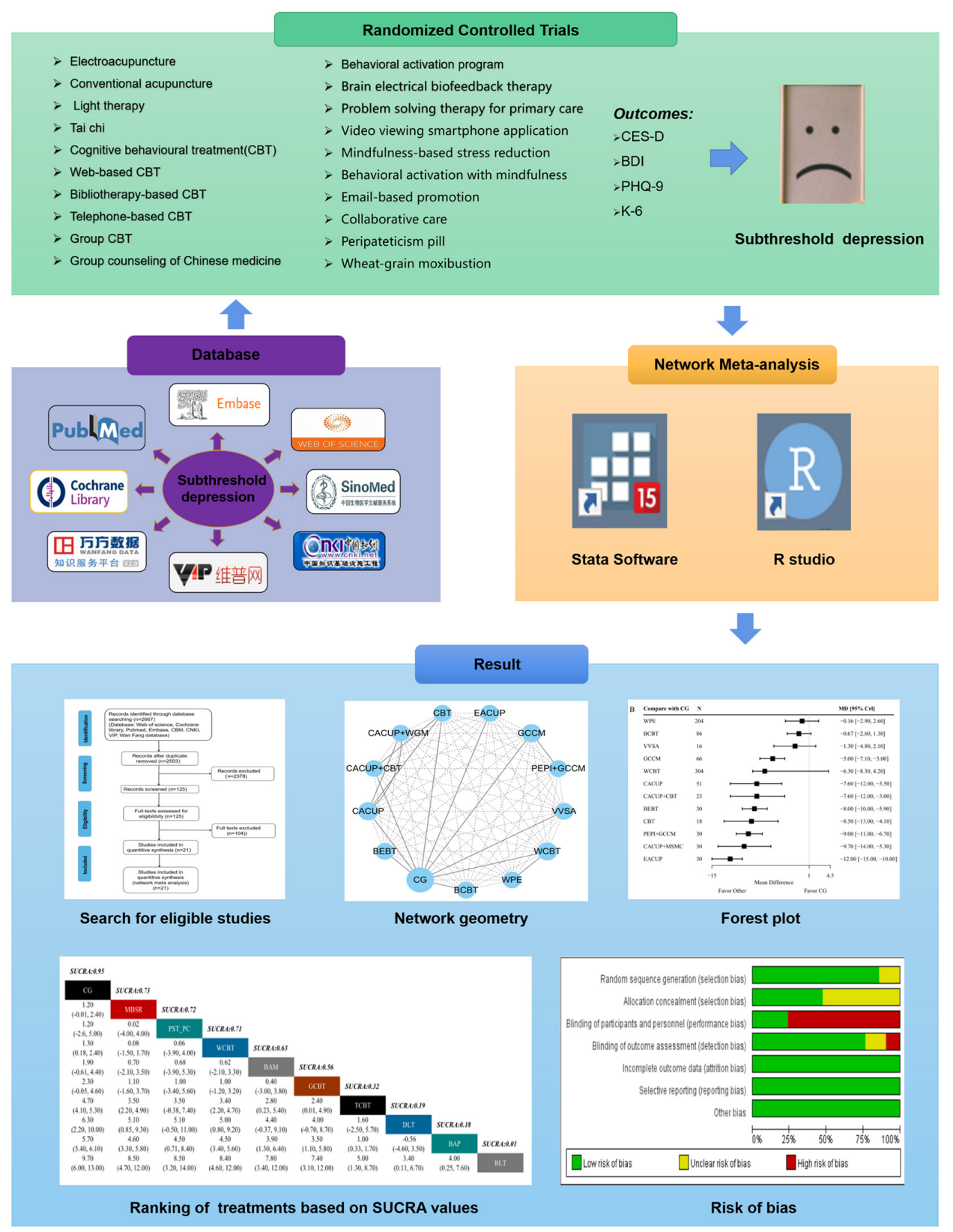

GRAPHICAL ABSTRACT | Graphical abstract of the network meta-analysis. Note: CES-D, Center for Epidemiologic Studies Depression Scale; BDI, Beck Depression Inventory Scale; PHQ-9, the nine-Item Patient Health Questionnaire; K6, Kessler Screening Scale for Psychological Distress; SUCRA, surface area under the cumulative ranking curves.

\section{INTRODUCTION}

Subthreshold depression (SD) is defined in the Diagnostic and Statistical Manual of Mental Disorders as including "dysthymia," "brief recurrent depression," and "minor depressive disorder" (Keller et al., 1995), but not meeting criteria for major depressive disorder (Pincus et al., 1999). Population-based studies have found that SD has a wide prevalence, about 1.4-17.2\% (Cuijpers and Smit, 2004), and the prevalence may be higher in the elderly or patients with chronic diseases (Cole and Dendukuri, 2003). Moreover, SD patients not only suffer from reduced quality of life, increased functional disability and mortality rate, but also require more service utilization and economic cost (Cuijpers and Smit, 2002; Cuijpers and Schoevers, 2004; Cuijpers et al., 2013). One third to one half of patients have moderate functional impairment, and at least $10-20 \%$ of patients have progressed to severe functional impairment at the 12-month follow-up (Jaffe et al., 1994; Kroenke, 2006; Lyness et al., 2006; Rodríguez et al., 2012). Studies have found that many patients with SD have persistent depressive symptoms, which is considered to be a risk factor for the development of major depressive disorder and other mental disorders (Cuijpers and Smit, 2004; Johnson et al., 2009). 
Thus, it is important to determine effective methods for the treatment of SD (Kroenke, 2017).

Early intervention may reduce the risk of symptom progression in patients with SD (Van't Veer-Tazelaar et al., 2011). There are various treatment strategies for SD, including psychotherapy, pharmacotherapy, exercise therapy, and traditional Chinese medicine (TCM) therapy. Previous studies showed that different types of psychotherapy, including cognitive behavior therapy and behavior activation therapy, could reduce the Beck Depression Inventory scale (BDI) score of adults with SD and reduce the incidence of major depressive disorder over 12 months (Furukawa et al., 2012; Buntrock et al., 2015; He et al., 2019). In one meta-analysis of 700 patients, psychotherapy was shown to be more beneficial to patients with SD than care-as-usual approaches and it may prevent the onset of severe depression (Cuijpers et al., 2007). However, psychotherapy is often not readily accessible due to long-cycle and high cost of treatment (Juul et al., 2019). Pharmacotherapy for SD focuses on the use of antidepressants such as tricyclic drugs, 5-hydroxytryptamine, reuptake inhibitors, and others. Antidepressants are one of the main therapies used for moderateto-severe depressive episodes, but one recent study found that antidepressants are often not better than the placebo for treating SD in randomized trials (Baumeister, 2012; Wang et al., 2019). Therefore, it still unclear whether antidepressants are suitable for the treatment of SD. Meanwhile, studies have confirmed that exercise, TCM psychotherapy, acupuncture, and moxibustion treatments are all effective in the treatment of $\mathrm{SD}$, but there is still a lack of valid and reliable evaluation for these therapies (Tan et al., 2014; Gou et al., 2017; Li et al., 2017a; Xie, 2018). So far, no comparison of the treatment efficacy has been conducted among the above different therapies, which limits decision-making for patients with SD in the clinic and the future research for treatment of SD.

Network meta-analysis of clinical trials involves multivariate and multilevel analysis, which allows clinicians to assess and rank the efficacy of different treatments based on both direct and indirect comparisons (Higgins and Whitehead, 1996; Salanti et al., 2008; Shim et al., 2017). The statistical methods used in network meta-analysis are mainly divided into those with frequentist and Bayesian frameworks (Jansen et al., 2011). The advantages of Bayesian methods over frequentist methods are the use of informative priors and the possibility of hierarchical modeling, which may allow more comprehensive use of the information from historical and vertical data (Yin et al., 2017). Therefore, we conducted a Bayesian network meta-analysis in this study to identify the efficacy of multiple therapies for SD and rank the efficacy of different interventions. The result will help clinicians to choose the optimum prescription from multiple treatments for SD in practice.

\section{MATERIALS AND METHODS}

\section{Search Strategy}

We searched the following databases: PubMed, Embase, Web of Science, Cochrane Central Register of Controlled Trials
(CENTRAL), Chinese Biomedical Literature Database (CBM), China National Knowledge Infrastructure (CNKI), Wan Fang database, and the China Science and Technology Journal database (VIP) that were available by April 3, 2021, using these keywords: "subthreshold depression" OR "subsyndromic depression” AND “randomized” OR “random.”

\section{Inclusion and Exclusion Criteria}

Studies were included according to the following criteria:

(1) Participants: patients included met the following criteria: they were diagnosed as SD based on the Diagnostic and Statistical Manual of Mental Disorders criteria (Sheehan et al., 1998) or Center for Epidemiologic Studies Depression Scale (CES-D) score $\geq 16$ (Shima et al., 1984), BDI score $\geq 14,7 \leq 17$-item Hamilton rating scale for depression score $<17$, or $8 \leq 24$-item Hamilton rating scale for depression score $<20$.

(2) Intervention: interventions in the treatment groups included acupuncture (electroacupuncture, conventional acupuncture), cognitive behavioral treatment (web-based cognitive behavioral treatment, bibliotherapy-based cognitive behavioral treatment, telephone-based cognitive behavioral therapy), video viewing smartphone application, behavioral activation with mindfulness, behavioral activation program, mindfulness-based stress reduction, problem solving therapy for primary care, email-based promotion, collaborative care, brain electrical biofeedback therapy, tai chi, bright light therapy, dim light therapy, group counseling with Chinese medicine, drug therapy (peripateticism pill), and moxibustion (wheat-grain moxibustion).

(3) Comparators: patients in the control group (CG) were the ones in a waiting list for treatment, under usual care, or having no treatments.

(4) Outcomes: outcomes in this study included at least one of the following evaluation indicators: CES-D, BDI, the 9-item patient health questionnaire (PHQ-9), or the Kessler screening scale for psychological distress (K-6).

(5) Types of studies that were included: randomized controlled trials (RCTs). The following studies were excluded: non-RCTs, single-arm design trials, conference abstracts, systematic reviews, or meta-analyses.

\section{Outcome Measures}

We used CES-D as the primary outcome, with higher CES$\mathrm{D}$ scores indicating more severe depressive symptoms. The secondary outcomes were BDI, PHQ-9, and K-6. The higher scores for BDI, PHQ-9 and K-6 indicate more severe depressive symptoms. The effect sizes for all outcomes were mean difference (MD) and 95\% credible intervals (CrI).

\section{Data Collection and Quality Assessment}

Two investigators independently scanned the titles and abstracts to confirm that the remaining studies met the predefined eligibility criteria, and full-text reviews were conducted for all potentially included studies. The data was extracted using a standard table, including information on the characteristics of the population, intervention(s), comparison(s), and outcome(s). The 
quality of each included trial was assessed by two authors based on the Cochrane Risk of Bias tool (Higgins et al., 2011).

\section{Statistical Analysis}

We conducted a Bayesian framework network meta-analysis by using $\mathrm{R}$ studio 4.0. The research data included in this network meta-analysis were evaluated by the Markov chain Monte Carlo method with 10,000 burn-ins and 50,000 iterations of four each chain and a thinning interval of 10 (Ades et al., 2006). A Brooks Gelman Rubin diagnostic plot was used to assess model convergence and the potential scale reduction parameter (PSRF) was used to evaluate the convergence of the results. When $1.00 \leq \mathrm{PSRF} \leq 1.05$, it indicates that the results have good convergence and high reliability (Van Valkenhoef et al., 2012). $\mathrm{I}^{2}$ statistic and its 95\% CrI were used to measure statistical heterogeneity, which is considered substantial when $I^{2}>50 \%$ (Higgins et al., 2003). Node-splitting analysis helps to determine the consistency test with an inconsistency model. In this study, a consistency model was chosen when the $p$-value of the node-splitting analysis was $>0.05$. If the $p$-value of the node-splitting analysis was $<0.05$, an inconsistency model was selected (Dias et al., 2010). We calculated the surface area under the cumulative ranking curves (SUCRA) to rank the curative effect of various interventions. The value range was 0-1. We used Begg's test and Egger's test to check publication bias, with a $p$-value of $<0.05$ indicating publication bias (Higgins et al., 2011). RevMan 5.3 software was used for bias risk assessment.

\section{RESULTS}

\section{Research Identification and Selection}

The literature search conducted retrieved 2,667 records. After deleting duplicates, 2,503 individual studies were recovered based on titles and abstracts. Among them, 125 studies were further selected for full-text review and 2,378 studies were removed due to the following reasons: non-SD, case report, animal models, republication, or review. Further examination excluded 104 studies according to the following reasons:

(1) The outcome was not the CES-D, BDI, PHQ-9, or K-6. (2) The studies were not RCTs. The remaining 21 studies were used for meta-analysis, including 5,048 patients (Figure 1). The characteristics of the selected trials are shown in Table $\mathbf{1 .}$

\section{Research Quality}

We used the Cochrane Risk of Bias Tool to assess the quality of the above selected 21 studies. As shown in Supplementary Figure 1, 18 studies (85\%) adopted a random sequence generation process using a computer random number generator or a random number table, 10 (47\%) described the use of allocation or concealment methods, 5 (23\%) described the blinding methods for researchers and participants, 16 (76\%) described the blinding methods of outcomes assessment. In terms of incomplete outcome data, selective reporting and other bias, all included studies are assessed as low risk.

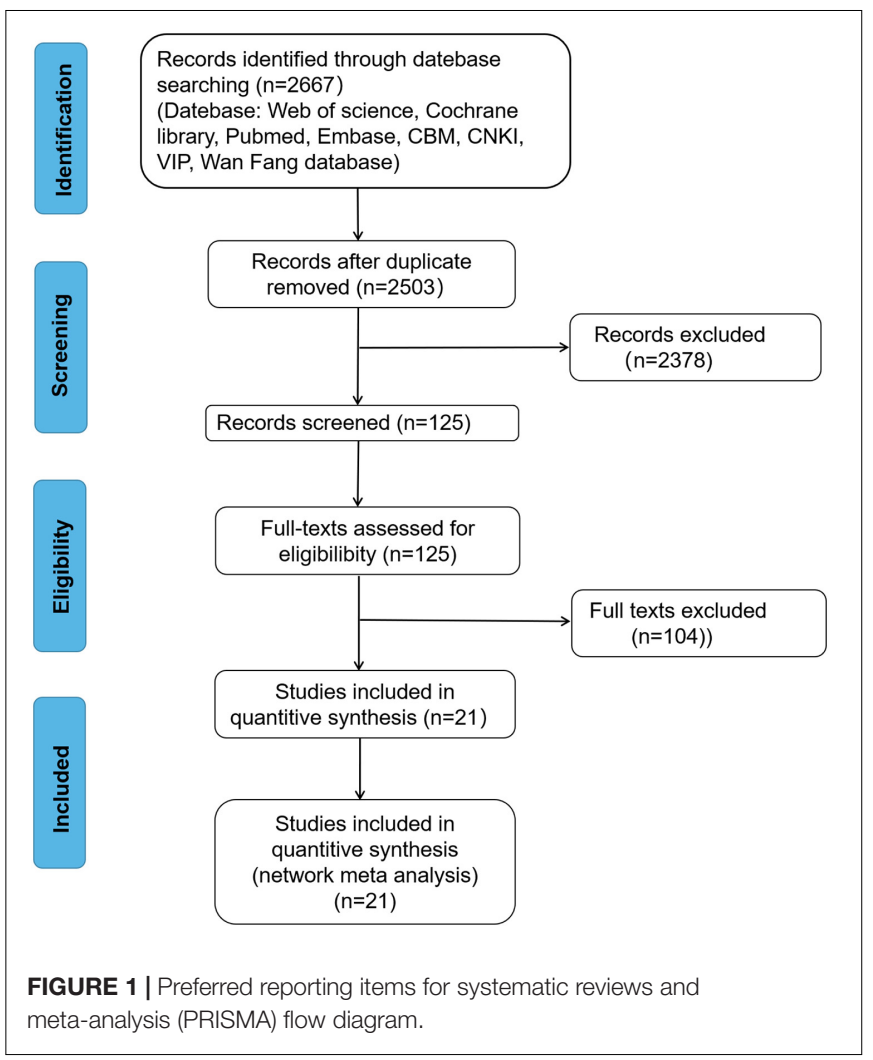

\section{Primary Outcome: Center for Epidemiologic Studies Depression Scale}

In the network meta-analysis for the CES-D, 10 trials and 13 interventions were included, and the network plot is shown in Figure 2A. A forest plot showing the result of CG compared with other interventions is presented in Figure 2B. We found that most of the treatments show better efficacy on CES-D compared with CG alone. These interventions include electroacupuncture ( $\mathrm{MD}-12.00$, 95\% CrI -15.00, -10.00), conventional acupuncture plus wheat-grain moxibustion (MD $-9.70,95 \% \mathrm{CrI}-14.00,-5.30)$, the peripateticism pill plus group counseling with Chinese medicine (MD -9.00, 95\% CrI -11.00, -6.70), cognitive behavioral treatment ( $\mathrm{MD}-8.50$, 95\% CrI $-13.00,-4.10)$, brain electrical biofeedback therapy instrument (MD -8.00, 95\% CrI -10.00, -5.90), conventional acupuncture plus cognitive behavioral treatment ( $\mathrm{MD}-7.60,95 \% \mathrm{CrI}$ $-12.00,-3.00)$, conventional acupuncture (MD $-7.60,95 \%$ CrI $-12.00,-3.50)$, group counseling with Chinese medicine (MD -5.00, 95\% CrI -7.10, -3.00). SUCRA values is used to rank the curative effect of various treatments. As shown in Figure 2C, the results showed that electroacupuncture (2\%) had the lowest SUCRA value across the various interventions, which means it had the highest probability of being the most effective treatment for CES-D. The following ranked treatments were conventional acupuncture plus wheat-grain moxibustion (16\%), the peripateticism pill plus group counseling with Chinese medicine (24\%), cognitive behavioral treatment (30\%), brain electrical biofeedback therapy instrument (34\%), 
TABLE 1Characteristic of included studies.

\begin{tabular}{|c|c|c|c|c|c|c|}
\hline Study ID & Samples size & Scanning of SD & $\begin{array}{l}\text { Treatment group } \\
\text { interventions }\end{array}$ & $\begin{array}{l}\text { Control group } \\
\text { interventions }\end{array}$ & Course of treatment & Outcome \\
\hline Tan, 2020 & 60 & $\begin{array}{l}\text { CES-D } \geq 16 \\
7 \leq \text { HAMD- } 17<17\end{array}$ & CACUP + WGM & CACUP & 4 weeks & CES-D \\
\hline Xie, 2018 & 60 & $\begin{array}{l}\text { CES-D } \geq 16 \\
7 \leq \mathrm{HAMD}-17<17\end{array}$ & EACUP & $\mathrm{CG}$ & 6 weeks & CES-D \\
\hline Li et al., 2017b & 60 & ZYYXH/T49-2008 & PEPI + GCCM & GCCM & 12 weeks & CES-D \\
\hline Zhang, 2015 & 79 & $\begin{array}{l}\text { CES-D } \geq 16 \\
7 \leq \text { HAMD- } 17<17\end{array}$ & $\begin{array}{l}\text { CACUP/CBT/CACUP + } \\
\text { CBT/CG }\end{array}$ & & 8 weeks/6 weeks & CES-D \\
\hline Dong et al., 2015 & 60 & CES-D > 16 & BEBT & $\mathrm{CG}$ & 3 weeks & CES-D \\
\hline Tan et al., 2014 & 72 & $7 \leq \mathrm{HAMD}-17<17$ & GCCM & CG & 8 weeks & CES-D \\
\hline Ebert et al., 2018 & 204 & CES-D $\geq 16$ & WCBT & CG & 12 weeks & CES-D \\
\hline Buntrock et al., 2015 & 406 & CES-D $\geq 16$ & WCBT & WPE & 3-6 weeks & CES-D \\
\hline Joling et al., 2011 & 170 & CES-D $\geq 16$ & BCBT & CG & 12 weeks & CES-D \\
\hline Kageyama et al., 2021 & 32 & CES-D $\geq 16$ & WSA & CG & 8 weeks & CES-D, K-6 \\
\hline Jiang et al., 2020 & 142 & $\begin{array}{l}\text { CES-D } \geq 16 \\
\text { BDI- } \| \geq 14\end{array}$ & BLT/DLT/CG & & 8 weeks & BDI-II \\
\hline Spek et al., 2007 & 301 & CES-D $\geq 12$ & WCBT/GCBT/CG & & 10 weeks & BDI-II \\
\hline Furukawa et al., 2012 & 118 & $\mathrm{BDI}-\| \geq 10 ; \mathrm{K}-6 \geq 9$ & TCBT & $\mathrm{CG}$ & 8 weeks & BDI-II, K-6, PHQ-9 \\
\hline Kasckow et al., 2014 & 23 & CES-D $\geq 11$ & PST-PC & $\mathrm{CG}$ & 6-8 weeks & BDI-II \\
\hline Zhang et al., 2019 & 56 & $\mathrm{BDI}>14 ; \mathrm{SDS}>53$ & MBSR & CG & 8 weeks & BDI-II \\
\hline Takagaki et al., 2018 & 118 & $\mathrm{BDI}-\| \geq 10$ & BAP & CG & 5 weeks & BDI-II \\
\hline Imamura et al., 2014 & 762 & NM & WCBT & CG & 6 weeks & BDI-II, K-6 \\
\hline Wong et al., 2018 & 231 & $5<\mathrm{PHQ}-9<9$ & BAM & CG & 8 weeks & BDI-II \\
\hline Xie, 2020 & 63 & CES-D $\geq 16$ & Tai Chi & CG & 12 weeks & PHQ-9 \\
\hline Morgan et al., 2012 & 1326 & $5<\mathrm{PHQ}-9<9$ & EBP & CG & 6 weeks & PHQ-9 \\
\hline Gilbody et al., 2017 & 705 & DSM-IV & $\mathrm{COC}$ & CG & 8 weeks & PHQ-9 \\
\hline
\end{tabular}

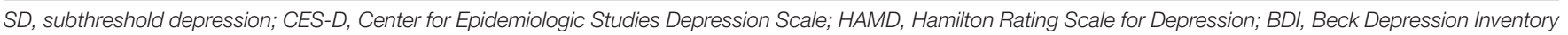

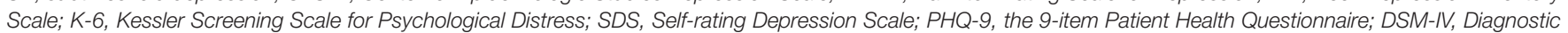

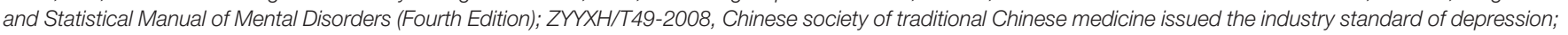

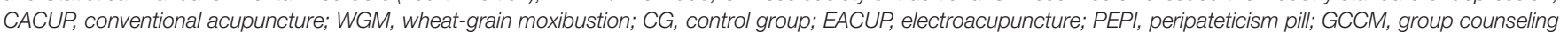

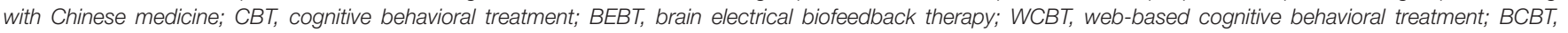

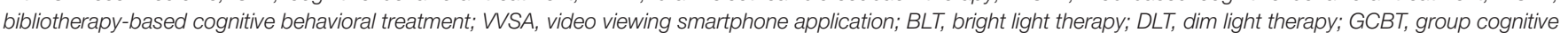

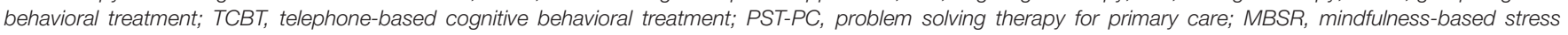

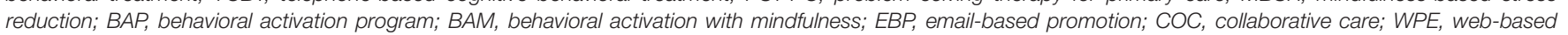
psycho education; NM, not mentioned.

conventional acupuncture plus cognitive behavioral treatment (40\%), conventional acupuncture (41\%), web-based cognitive behavioral treatment (52\%), group counseling with Chinese medicine $(62 \%)$, video viewing smartphone application (82\%), bibliotherapy-based cognitive behavioral treatment $(85 \%)$, and web-based psycho-education (90\%).

\section{Secondary Outcome: Beck Depression Inventory Scale}

Eight trials and ten interventions were involved in measuring BDI outcome. The network geometry is presented in Figure 3A. From the forest plot of Figure 3B, we found that bright light therapy (MD -9.70, 95\% CrI $-13.00,-6.00)$, behavioral activation program (MD $-5.70,95 \% \mathrm{CrI}-6.10,-5.40)$, dim light therapy (MD -6.30, 95\% CrI -10.00, -2.20), telephone-based cognitive behavioral treatment (MD $-4.70,95 \% \mathrm{CrI}-5.30,-4.10)$, group cognitive behavior treatment (MD -2.30, 95\% CrI -4.60, 0.10), behavioral activation with mindfulness (MD -1.90 , 95\% CrI $-4.40,0.61$ ), web-based cognitive behavioral treatment (MD
$-1.30,95 \%$ CrI $-2.40,-0.18)$, and mindfulness-based stress reduction ( $\mathrm{MD}-1.20,95 \% \mathrm{CrI}-2.40,0.03$ ) had better efficacy compared with CG alone on BDI. As shown in Figure 3C, Bright light therapy (1\%) showed the lowest SUCRA value in all treatments, which indicates that it had the highest probability of being the most effective treatment for BDI for SD. The following ranked treatments were behavioral activation program (18\%), dim light therapy (19\%), telephone-based cognitive behavioral treatment (32\%), group cognitive behavior treatment (56\%), behavioral activation with mindfulness (63\%), webbased cognitive behavioral treatment (71\%), problem solving therapy for primary care $(72 \%)$, and mindfulness-based stress reduction $(73 \%)$.

\section{Secondary Outcome: Patient Health Questionnaire-9}

To evaluate the PHQ-9 outcome, three studies and four interventions were included. The network plot is shown in Figure 4A. As shown in the forest plot of PHQ-9 (Figure 4B), 
A

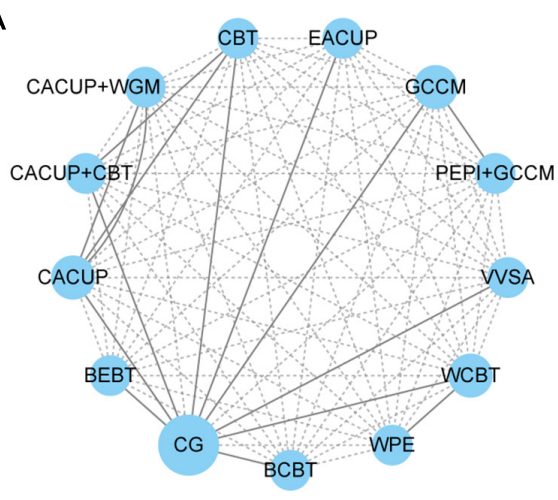

C

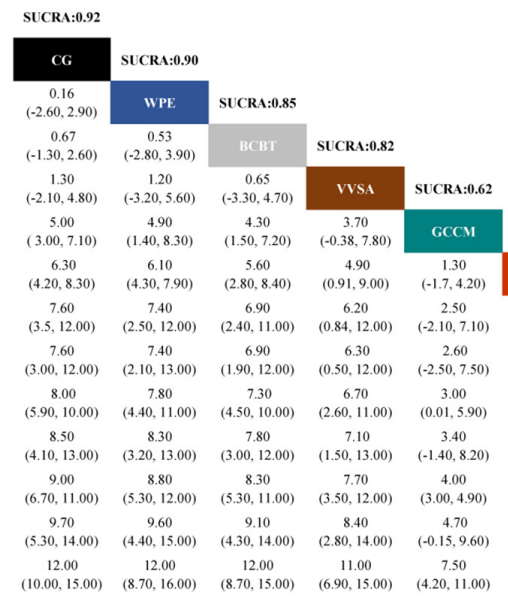

B

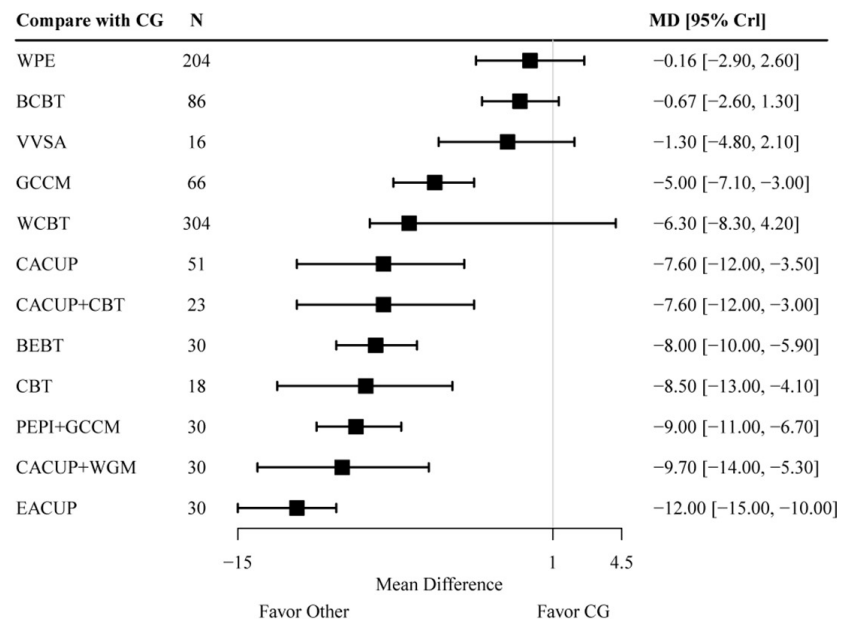

SUCRA:0.52

WCBT SUCRA:0.41

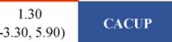

SICRA:0.40

\begin{tabular}{cc|c|c}
1.30 & 0.03 & CACUP+CBT & SUCRA: 0.34 \\
$(-3.70,6.20)$ & $(-3.20,3.20)$ & &
\end{tabular}

$1.70 \quad 0.43$

$\begin{array}{ccc}1.70 & 0.43 & 0.43 \\ -.20,4.60) & (-4.10,5.00) & (-4.50,5.40)\end{array}$

2.20

$(-2.10,3.80)$

$(-0.35,5.80)$

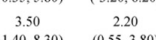

$(0.140,8.30) \quad(0.55,3.80)$

$\begin{array}{cc}6.20 & 5.00 \\ (300.9 .40) & (0.22,9.70)\end{array}$

$0.88 \quad$ SUCRA:0.30

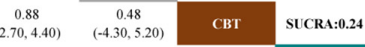

$1.40 \quad 1.00$

$\begin{array}{ccc}1.00 & 0.52 & \text { PEPI+GCCM } \\ (-2.10,4.10) & (-430,5.50) & \text { PEP } \\ 1.80 & 1.30 & 0.77\end{array}$

$(-2.10,4.10) \quad(-4.30,5.50) \quad$ PEPI+GCCM SUCRA:0.16

2.20

$50,5.70$

4.90

$(-3.100 .660)$

$(-3.10,6.60)$

\begin{tabular}{cc|c}
1.30 & 0.77 \\
$(-2.10,4.70)$ & $(-4.20,5.70)$ & CACUP+WGM \\
& 3.50 &
\end{tabular}

$(-2.10,4.70) \quad(-4.20,5.70)$

$\begin{array}{cc}4.00 & 3.50 \\ (-0.90,9.00) & (0.14,6.90)\end{array}$

2.80
$(-220,780)$

EACUP

FIGURE 2 | Results of the network meta-analysis for CES-D. (A) Network geometry of eligible comparisons for CES-D. (B) Forest plot of the network meta-analysis compared with control group. (C) Ranking of each intervention based on the SUCRA values and the league table for the relative effects of all treatments. EACUP, electroacupuncture; CACUP, conventional acupuncture; WGM, wheat-grain moxibustion; GCCM, group counseling with Chinese medicine; CBT, cognitive behavioral treatment; BEBT, brain electrical biofeedback therapy; WCBT, web-based cognitive behavioral treatment; WSA, video viewing smartphone application; BCBT, bibliotherapy-based cognitive behavioral treatment; WPE, web-based psycho education; CG, control group; SUCRA, surface under the cumulative ranking curve; MD, weighted mean difference; Crl, credible interval.

tai chi (MD -3.00, 95\% CrI -4.00, -2.00), collaborative care (MD - 1.30, 95\% CrI - 1.40, - 1.20), and email-based promotion (MD - $0.8095 \%$ CrI $-1.30,-0.27$ ) showed better efficacy in PHQ-9 compared with CG alone. As shown in Figure 4C, tai chi $(0 \%)$ had the lowest SUCRA value of the four interventions, which indicated that it had the highest probability of being the most effective treatment for PHQ-9 for SD. Other following effective treatments were collaborative care (34\%) and emailbased promotion (66\%).

\section{Secondary Outcome: Kessler Screening Scale for Psychological Distress}

There were three trials and four interventions that measured a K-6 outcome. The network plot is presented in Figure 5A. As indicated in the forest plot for K-6 (Figure 5B), telephonebased cognitive behavioral treatment (MD -2.50 95\% CrI -2.70, -2.30) had better efficacy compared with CG alone. As shown in Figure 5C, telephone-based cognitive behavioral treatment (0\%) had the lowest SUCRA values among the four interventions, which indicated that it had the highest probability of being the most effective treatment for K-6. The outcome from other two treatments were web-based cognitive behavioral treatment (60\%) and video viewing smartphone application (62\%), respectively.

\section{Model Convergence, Heterogeneity, and Publication Bias}

The shrinking factors in the Brooks Gelman Rubin diagnostic plots for all outcomes were less than 1.05 (Supplementary Figure 2). The statistical heterogeneity for all outcomes were low $\left(I^{2}<50 \%\right.$, ranging from $5 \%$ to $\left.17 \%\right)$. According to results of Begg's and Egger's tests with a funnel plot, no publication bias was detected $(p>0.05)$ in our study (Supplementary Figure 3 and Supplementary Table 1). There was no obvious inconsistency shown in this network meta-analysis. 

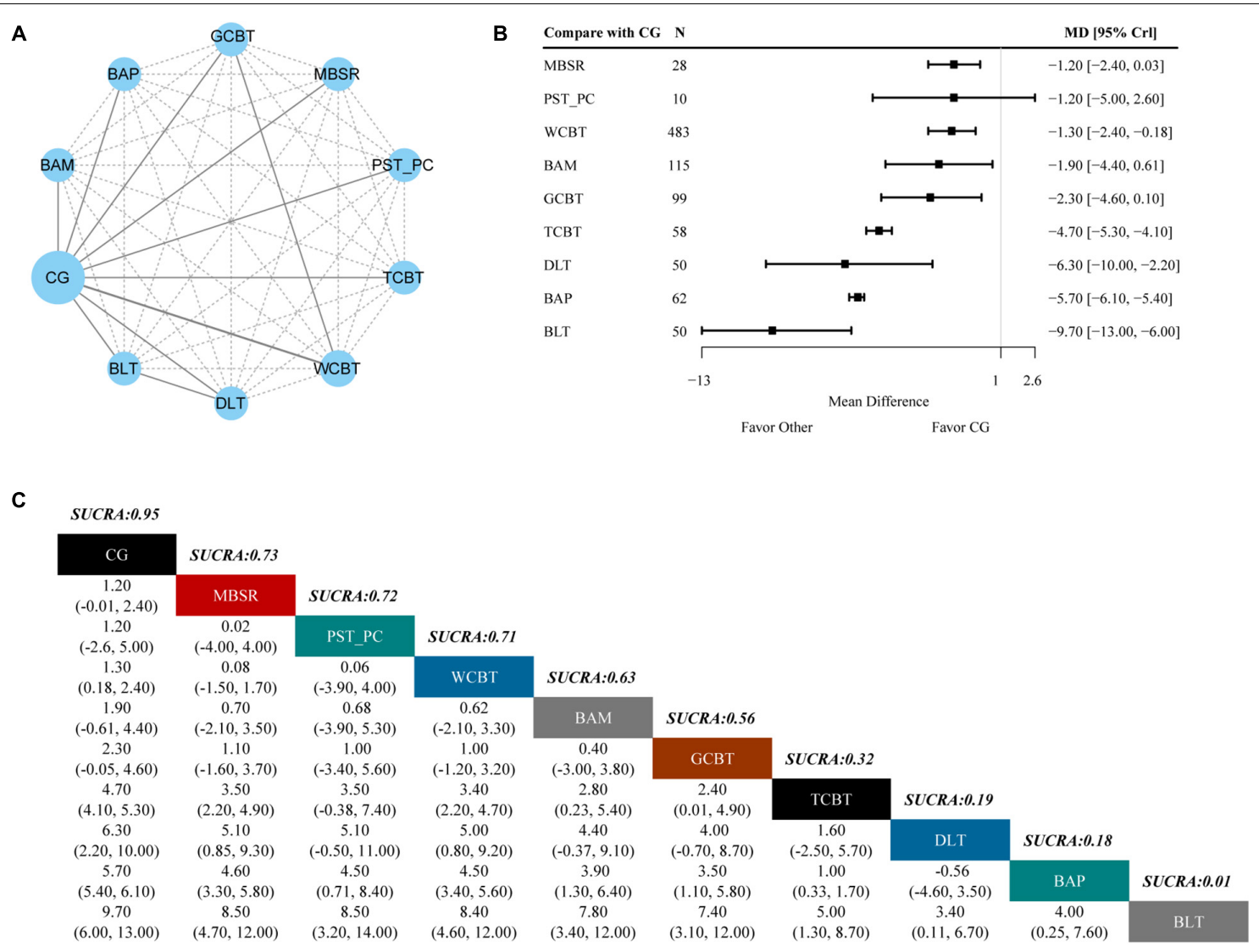

FIGURE 3 | Results of the network meta-analysis for BDI. (A) Network geometry of eligible comparisons for BDI. (B) Forest plot of the network meta-analysis compared with control group. (C) Ranking of each intervention based on the SUCRA values and the league table for the relative effects of all treatments. BLT, bright light therapy; DLT, dim light therapy; BAP, behavioral activation program; TCBT, telephone-based cognitive behavioral treatment; GCBT, group cognitive behavioral treatment; BAM, behavioral activation with mindfulness; WCBT, web-based cognitive behavioral treatment; PST-PC, problem solving therapy for primary care; MBSR, mindfulness-based stress reduction; CG, control group; SUCRA, surface under the cumulative ranking curve; MD, weighted mean difference; Crl, credible interval.

\section{DISCUSSION}

In this network meta-analysis, our results provided evidence that all the involved treatments for patients with SD showed advantages over waiting for treatment or no treatment. Furthermore, we found that electroacupuncture was the best therapy to improve outcome on the CES-D. Meanwhile, bright light therapy was the optimum treatment for the outcome on the BDI and tai chi was best effective in promoting outcome on the PHQ-9. However, telephone-based cognitive behavioral treatment was the best intervention for outcome on the K-6.

Electroacupuncture has been widely used to treat psychiatric conditions including depression (Woo et al., 2015), which may be associated with multiple mechanisms. First, relevant research found that the serotonin system plays an important role in the pathophysiology of depression (Mouri et al., 2016). Electroacupuncture was reported to restore hippocampal CA1 synaptic plasticity by regulating the level of serotonin system receptors, thereby improving depression-like behavior (Han et al., 2018). Second, it has been found that proinflammatory cytokines play an important role in neurogenesis and neuroprotection (Kim et al., 2016). Patients with depression have high levels of pro-inflammatory cytokines, acute phase proteins, chemokines, and cell adhesion molecules (Raison et al., 2006). Electroacupuncture reduced hippocampal neuroinflammation in depressed rats by reducing the expression of pyrin domain-containing protein 3 inflammatory components (ASC and caspase-1), activating microglia and ATP gated transmembrane protein $(\mathrm{P} 2 \times 7)$ receptor as well as reducing levels of interleukin-1 beta (IL-1 $\beta$ ), IL-18, IL-6, and tumor necrosis factor alpha (Yue et al., 2018; Li et al., 2021). Furthermore, hypothalamic pituitary adrenal axis dysregulation, which is generally considered a diagnostic criterion for early stages of depression, is also implicated in the pathology of depression (Du and Pang, 2015). Studies have shown that electroacupuncture could exert anti-depressive activity by regulating the hypothalamic pituitary adrenal axis (Le et al., 2016).

Consistent with our results, light therapy has been widely used to treat seasonal depression (Avissar et al., 1999). Light can affect mood and learning through different retinal brain pathways (Fernandez et al., 2018). The antidepressant effects of light therapy have been found to be related to changes in neurotransmitters, including serotonin and catecholamine 
A

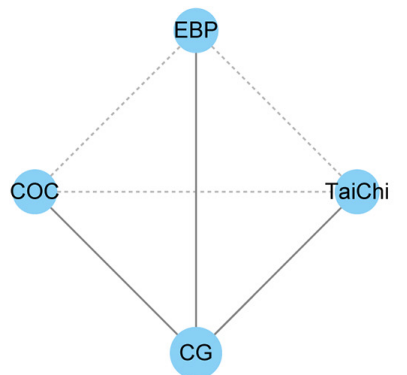

C

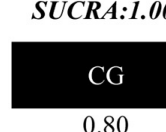

0.80

$(0.27,1.30)$

1.30

$(1.20,1.40)$

3.00

$(2.00,4.00)$
B

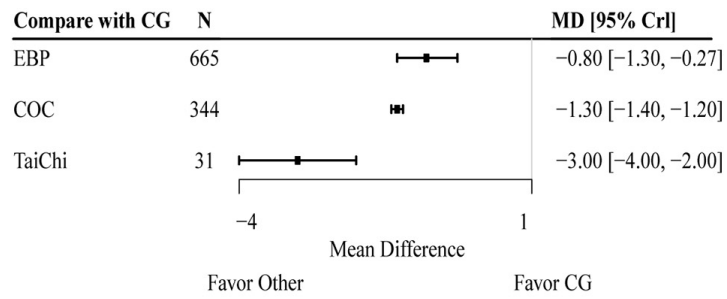

FIGURE 4 | Results of the network meta-analysis for PHQ-9. (A) Network geometry of eligible comparisons for PHQ-9. (B) Forest plot of the network meta-analysis compared with control group. (C) Ranking of each intervention based on the SUCRA values and the league table for the relative effects of all treatments. EBP, email-based promotion; COC, collaborative care; CG, control group; SUCRA, surface under the cumulative ranking curve; MD, weighted mean difference; Crl, credible interval.

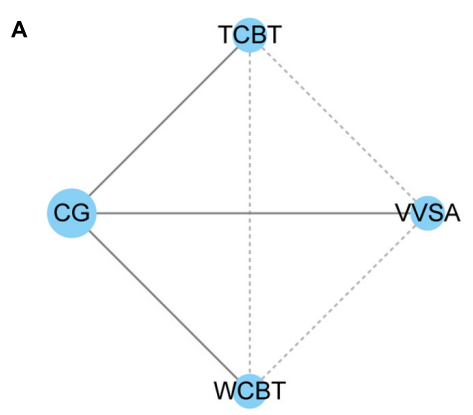

SUCRA:0.78

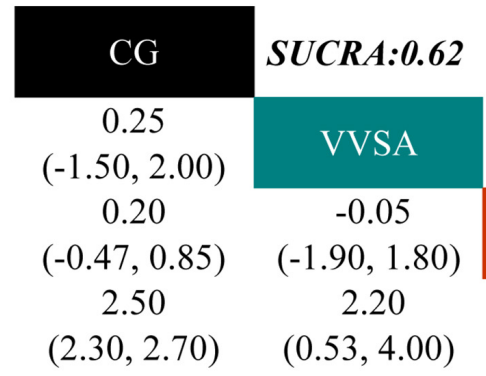

B

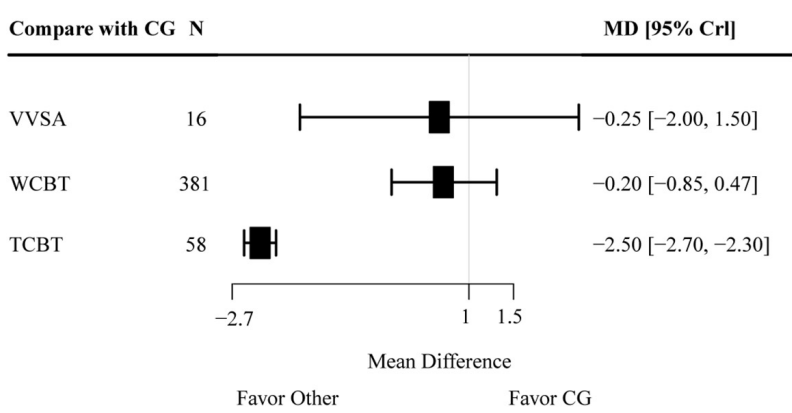

Favor Other

Favor CG

FIGURE $\mathbf{5}$ | Results of the network meta-analysis for K-6. (A) Network geometry of eligible comparisons for K-6. (B) Forest plot of the network meta-analysis compared with control group. (C) Ranking of each intervention based on the SUCRA values and the league table for the relative effects of all treatments. TCBT, telephone-based cognitive behavioral treatment; WCBT, web-based cognitive behavioral treatment; WSA, video viewing smartphone application; CG, control group; SUCRA, surface under the cumulative ranking curve; MD, weighted mean difference; Crl, credible interval. 
(Lam et al., 1996; Neumeister, 2003). Previous studies also found that light therapy could synchronize the biological clock with the circadian rhythm of the environment, which is considered to be closely related to depression (Lam and Levitan, 2000; Pail et al., 2011; Jagannath et al., 2013). Furthermore, light therapy has the advantages of low cost, high security, and direct availability (Bais et al., 2016). Together, all these studies provide further evidence that light therapy may be the optimal treatment for patients with SD.

As an adjuvant therapy for depression, physical exercise has attracted increasing attention (Legrand and Neff, 2016). One study found that aerobic exercise can increase the levels of brain-derived neurotrophic factor, which has been shown to decrease in individuals with severe depression (BocchioChiavetto et al., 2010). Tai chi was reported to ameliorate the depressive symptoms of elderly Chinese patients (Cho, 2008). Meanwhile, other studies provide evidences that tai chi could regulate brain networks associated with depression and alleviate depressive symptoms by regulating cortisol levels and immune system (Walther et al., 2018; Kong et al., 2019). However, given that the sample sizes of clinical research studies were small, whether tai chi has a unique benefit for treatment of SD over other physical exercise requires further study.

Our results suggested that telephone-based cognitive behavioral treatment is the best treatment for improving K-6, which is used to screen for mental health problems and measure the severity of the impact of these problems (Mitchell and Beals, 2011). Telephone-based cognitive behavioral treatment is a novel method for the treatment of SD (Furukawa et al., 2012). It may overcome some of the obstacles preventing patients from receiving traditional psychotherapy services, including occupational or social constraints, residency in underserved areas, and the need to commute, and therefore may be a more appropriate choice in these situations (Bee et al., 2008). Taken together, our findings suggest that more attention should be paid to the application of telephone-based cognitive behavioral treatment.

There were some limitations in our study. First, many RCT studies did not include PHQ-9 and K-6, which limited the comprehensive evaluation of the efficacy on the two outcomes. Second, parts of the quality assessment risk were unsatisfactory because of the characteristics of waiting for treatment or no treatment, it hard to blind both therapist and participants. Finally, given that the number of patients included was relatively small, more multi-center and high-quality RCTs are needed in the future to validate our findings.

\section{REFERENCES}

Ades, A. E., Sculpher, M., Sutton, A., Abrams, K., Cooper, N., Welton, N., et al. (2006). Bayesian methods for evidence synthesis in cost-effectiveness analysis. Pharmacoeconomics 24, 1-19. doi: 10.2165/00019053-20062401000001

Avissar, S., Schreiber, G., Nechamkin, Y., Neuhaus, I., Lam, G. K., Schwartz, P. et al. (1999). The effects of seasons and light therapy on G protein levels in mononuclear leukocytes of patients with seasonal affective disorder. Arch. Gen. Psychiatry 56, 178-183. doi: 10.1001/archpsyc.56.2.178

\section{CONCLUSION}

The results of this comprehensive network meta-analysis provides a complete evaluation of currently available therapies for patients with SD. Our results suggest that electroacupuncture or bright light therapy may be the preferred selection in the treatment of SD. This study provide new insights into clinical treatment selection and may help the development of guidelines for the management of SD.

\section{DATA AVAILABILITY STATEMENT}

The original contributions presented in the study are included in the article/Supplementary Material, further inquiries can be directed to the corresponding author.

\section{AUTHOR CONTRIBUTIONS}

YJC and XJ designed this network meta-analysis. XJ, YL, YWC, JY, and SH collected the data. XJ, YX, XW, YL, and YWC analyzed the data. XJ, LY, FW, and TW performed the analysis. XJ and YJC wrote the manuscript. All authors contributed to writing of this manuscript and approved the final version of the manuscript.

\section{FUNDING}

This work was supported by National Key R\&D Program of China (2019YFC1712105). This work was also supported, in part, by the Science and Technology Program of Guangdong (2018B030334001); the National Natural Science Foundation of China (No. 81973948); Guangdong Province Universities and Colleges Pearl River Scholar Funded Scheme (2016); Innovation Team Program of Guangdong Provincial Department of Education (No. 2018KCXTD006); and National Natural Science Foundation of Guangdong Province (No. 2020ZDZX1059).

\section{SUPPLEMENTARY MATERIAL}

The Supplementary Material for this article can be found online at: https://www.frontiersin.org/articles/10.3389/fnbeh. 2021.755547/full\#supplementary-material

Bais, B., Kamperman, A. M., Van der Zwaag, M. D., Dieleman, G. C., Harmsen van der Vliet-Torij, H. W., Bijma, H. H., et al. (2016). Bright light therapy in pregnant women with major depressive disorder: study protocol for a randomized, double-blind, controlled clinical trial. BMC Psychiatry 16:381. doi: 10.1186/s12888-016-1092-2

Baumeister, H. (2012). Inappropriate prescriptions of antidepressant drugs in patients with subthreshold to mild depression: time for the evidence to become practice. J. Affect. Disord. 139, 240-243. doi: 10.1016/j.jad.2011.05.025

Bee, P. E., Bower, P., Lovell, K., Gilbody, S., Richards, D., Gask, L., et al. (2008). Psychotherapy mediated by remote communication technologies: 
a meta-analytic review. BMC Psychiatry 8:60 doi: 10.1186/1471244X-8-60

Bocchio-Chiavetto, L., Bagnardi, V., Zanardini, R., Molteni, R., Nielsen, M. G., Placentino, A., et al. (2010). Serum and plasma BDNF levels in major depression: a replication study and meta-analyses. World J. Biol. Psychiatry 11, 763-773. doi: 10.3109/15622971003611319

Buntrock, C., Ebert, D., Lehr, D., Riper, H., Smit, F., Cuijpers, P., et al. (2015). Effectiveness of a web-based cognitive behavioural intervention for subthreshold depression: pragmatic randomised controlled trial. Psychother. Psychosom. 84, 348-358. doi: 10.1159/000438673

Cho, K. L. (2008). Effect of Tai Chi on depressive symptoms amongst Chinese older patients with major depression: the role of social support. Med. Sport Sci. 52, 146-154. doi: 10.1159/000134295

Cole, M. G., and Dendukuri, N. (2003). Risk factors for depression among elderly community subjects: a systematic review and meta-analysis. Am. J. Psychiatry 160, 1147-1156. doi: 10.1176/appi.ajp.160.6.1147

Cuijpers, P., and Schoevers, R. A. (2004). Increased mortality in depressive disorders: a review. Curr. Psychiatry Rep. 6, 430-437. doi: 10.1007/s11920-0040007-y

Cuijpers, P., and Smit, F. (2002). Excess mortality in depression: a meta-analysis of community studies. J. Affect. Disord. 72, 227-236. doi: 10.1016/s0165-0327(01) 00413-x

Cuijpers, P., and Smit, F. (2004). Subthreshold depression as a risk indicator for major depressive disorder: a systematic review of prospective studies. Acta Psychiatr. Scand. 109, 325-331. doi: 10.1111/j.1600-0447.2004.00 301.x

Cuijpers, P., Smit, F., and van Straten, A. (2007). Psychological treatments of subthreshold depression: a meta-analytic review. Acta Psychiatr. Scand. 115, 434-441. doi: 10.1111/j.1600-0447.2007.00998.x

Cuijpers, P., Vogelzangs, N., Twisk, J., Kleiboer, A., Li, J., and Penninx, B. W. (2013). Differential mortality rates in major and subthreshold depression: metaanalysis of studies that measured both. Br. J. Psychiatry 202, 22-27. doi: 10.1192/ bjp.bp.112.112169

Dias, S., Welton, N. J., Caldwell, D. M., and Ades, A. E. (2010). Checking consistency in mixed treatment comparison meta-analysis. Stat. Med. 29, 932944. doi: 10.1002/sim.3767

Dong, C., Zhang, R. F., Liu, J. C., Wang, Q., Sun, H. D., and Zhang, A. R. (2015). Prognosis and $\mathrm{P} 3 \mathrm{~b}$ changes of 60 patients with subthreshold depression after EEG biofeedback intervention. J. Milit. Surg. South Chin. 17, 386-389.

Du, X., and Pang, T. Y. (2015). Is Dysregulation of the HPA-Axis a Core Pathophysiology Mediating Co-Morbid Depression in Neurodegenerative Diseases? Front. Psychiatry 6:32. doi: 10.3389/fpsyt.2015.00032

Ebert, D. D., Buntrock, C., Lehr, D., Smit, F., Riper, H., Baumeister, H., et al. (2018). Effectiveness of web-and mobile-based treatment of subthreshold depression with adherence-focused guidance: a single-blind randomized controlled trial. Behav. Ther. 49, 71-83. doi: 10.1016/j.beth.2017.05.004

Fernandez, D. C., Fogerson, P. M., Lazzerini Ospri, L., Thomsen, M. B., Layne, R. M., Severin, D., et al. (2018). Light Affects Mood and Learning through Distinct Retina-Brain Pathways. Cell 175, 71.e-84.e. doi: 10.1016/j.cell.2018. 08.004

Furukawa, T. A., Horikoshi, M., Kawakami, N., Kadota, M., Sasaki, M., Sekiya, Y., et al. (2012). Telephone cognitive-behavioral therapy for subthreshold depression and presenteeism in workplace: a randomized controlled trial. PLoS One 7:e35330. doi: 10.1371/journal.pone.0035330

Gilbody, S., Lewis, H., Adamson, J., Atherton, K., Bailey, D., Birtwistle, J., et al. (2017). Effect of collaborative care vs usual care on depressive symptoms in older adults with subthreshold depression: the CASPER randomized clinical trial. JAMA 317, 728-737. doi: 10.1001/jama.2017.0130

Gou, S. L., Zhu, C. J., Xu, F., and Tang, Q. S. (2017). Clinical observation on the intervention of tonifying kidney and regulating qi in subthreshold depression patients with kidney deficiency and liver depression. Beijing J. TCM. 36, 272274. doi: 10.16025/j.1674-1307.2017.03.021

Han, X., Wu, H., Yin, P., Chen, Z., Cao, X., Duan, Y., et al. (2018). Electroacupuncture restores hippocampal synaptic plasticity via modulation of 5-HT receptors in a rat model of depression. Brain Res. Bull. 139, 256-262. doi: 10.1016/j.brainresbull.2018.03.004

He, H. L., Zhang, M., Gu, C. Z., Xue, R. R., Liu, H. X., Gao, C. F., et al. (2019). Effect of Cognitive Behavioral Therapy on Improving the Cognitive Function in Major and Minor Depression. J. Nerv. Ment. Dis. 207, 232-238. doi: 10.1097/ NMD.0000000000000954

Higgins, J. P., Altman, D. G., Gøtzsche, P. C., Jüni, P., Moher, D., Oxman, A. D., et al. (2011). The cochrane collaboration's tool for assessing risk of bias in randomised trials. BMJ 343:d5928. doi: 10.1136/bmj.d5928

Higgins, J. P., Thompson, S. G., Deeks, J. J., Altman, D. G. (2003). Measuring inconsistency in meta-analyses. BMJ 327, 557-560. doi: 10.1136/bmj.327.7414. 557

Higgins, J. P., and Whitehead, A. (1996). Borrowing strength from external trials in a meta-analysis. Stat. Med. 15, 2733-2749. doi: 10.1002/(SICI)10970258(199612 30)15:24<2733:AID-SIM562>3.0.CO;2-0

Imamura, K., Kawakami, N., Furukawa, T. A., Matsuyama, Y., Shimazu, A., Umanodan, R., et al. (2014). Effects of an Internet-based cognitive behavioral therapy (iCBT) program in Manga format on improving subthreshold depressive symptoms among healthy workers: a randomized controlled trial. PLoS One 9:e97167. doi: 10.1371/journal.pone.0097167

Jaffe, A., Froom, J., and Galambos, N. (1994). Minor depression and functional impairment. Arch. Fam. Med. 3, 1081-1086. doi: 10.1001/archfami.3.12.1081

Jagannath, A., Peirson, S. N., and Foster, R. G. (2013). Sleep and circadian rhythm disruption in neuropsychiatric illness. Curr. Opin. Neurobiol. 23, 888-894. doi: 10.1016/j.conb.2013.03.008

Jansen, J. P., Fleurence, R., Devine, B., Itzler, R., Barrett, A., Hawkins, N., et al. (2011). Interpreting indirect treatment comparisons and network meta-analysis for health-care decision making: report of the ISPOR Task Force on Indirect Treatment Comparisons Good Research Practices: part 1. Value Health. 14, 417-428. doi: 10.1016/j.jval.2011.04.002

Jiang, L., Zhang, S., Wang, Y., So, K. F., Ren, C., and Tao, Q. (2020). Efficacy of light therapy for a college student sample with non-seasonal subthreshold depression: An RCT study. J. Affect. Disord. 2020, 443-449. doi: 10.1016/j.jad. 2020.08.055

Johnson, J. G., Cohen, P., and Kasen, S. (2009). Minor depression during adolescence and mental health outcomes during adulthood. Br. J. Psychiatry 195, 264-265. doi: 10.1192/bjp.bp.108.054239

Joling, K. J., van Hout, H. P., van't Veer-Tazelaar, P. J., van der Horst, H. E., Cuijpers, P., van de Ven, P. M., et al. (2011). How effective is bibliotherapy for very old adults with subthreshold depression? A randomized controlled trial. Am. J. Geriatr. Psychiatry 19, 256-265. doi: 10.1097/JGP.0b013e3181ec8859

Juul, S., Poulsen, S., Lunn, S., Sørensen, P., Jakobsen, J. C., and Simonsen, S. (2019). Short-term versus long-term psychotherapy for adult psychiatric disorders: a protocol for a systematic review with meta-analysis and trial sequential analysis. Syst. Rev. 8:169. doi: 10.1186/s13643-019-1099-0

Kageyama, K., Kato, Y., Mesaki, T., Uchida, H., Takahashi, K., Marume, R., et al. (2021). Effects of video viewing smartphone application intervention involving positive word stimulation in people with subthreshold depression: A pilot randomized controlled trial. J Affect Disord. 2021, 74-81. doi: 10.1016/j.jad. 2020.12.104

Kasckow, J., Klaus, J., Morse, J., Oslin, D., Luther, J., Fox, L., et al. (2014). Using problem solving therapy to treat veterans with subsyndromal depression: a pilot study. Int. J. Geriatr. Psychiatry 29, 1255-1261. doi: 10.1002/gps.4105

Keller, M. B., Klein, D. N., Hirschfeld, R. M., Kocsis, J. H., McCullough, J. P., Miller, I., et al. (1995). Results of the DSM-IV mood disorders field trial. Am. J. Psychiatry 152, 843-849. doi: 10.1176/ajp.152.6.843

Kim, Y. K., Na, K. S., Myint, A. M., and Leonard, B. E. (2016). The role of pro-inflammatory cytokines in neuroinflammation, neurogenesis and the neuroendocrine system in major depression. Prog. Neuropsychopharmacol. Biol. Psychiatry 64, 277-284. doi: 10.1016/j.pnpbp.2015.06.008

Kong, J., Wilson, G., Park, J., Pereira, K., Walpole, C., and Yeung, A. (2019). Treating Depression With Tai Chi: State of the Art and Future Perspectives. Front. Psychiatry 10:237. doi: 10.3389/fpsyt.2019.00237

Kroenke, K. (2006). Minor depression: midway between major depression and euthymia. Ann. Intern. Med. 144, 528-530. doi: 10.7326/0003-4819-144-7200604040-00013

Kroenke, K. (2017). When and How to Treat Subthreshold Depression. JAMA 317, 702-704. doi: 10.1001/jama.2017.0233

Lam, R. W., and Levitan, R. D. (2000). Pathophysiology of seasonal affective disorder: a review. Psychiatry Neurosci. 25, 469-480.

Lam, R. W., Zis, A. P., Grewal, A., Delgado, P. L., Charney, D. S., and Krystal, J. H. (1996). Effects of rapid tryptophan depletion in patients with seasonal 
affective disorder in remission after light therapy. Arch. Gen. Psychiatry 53, 41-44. doi: 10.1001/archpsyc.1996.01830010043007

Le, J. J., Yi, T., Qi, L., Li, J., Shao, L., and Dong, J. C. (2016). Electroacupuncture regulate hypothalamic-pituitary-adrenal axis and enhance hippocampal serotonin system in a rat model of depression. Neurosci. Lett. 615, 66-71. doi: 10.1016/j.neulet.2016.01.004

Legrand, F. D., and Neff, E. M. (2016). Efficacy of exercise as an adjunct treatment for clinically depressed inpatients during the initial stages of antidepressant pharmacotherapy: An open randomized controlled trial. J. Affect. Disord. 191, 139-144. doi: 10.1016/j.jad.2015.11.047

Li, K., Shi, G., Zhao, Y., Chen, Y., Gao, J., Yao, L., et al. (2021). Electroacupuncture Ameliorates Neuroinflammation-Mediated Cognitive Deficits through Inhibition of NLRP3 in Presenilin1/2 Conditional Double Knockout Mice. Neural. Plast. 2021:8814616. doi: 10.1155/2021/8814616

Li, S., Xu, X. Y., Guo, X. Z., and Xiao, Y. L. (2017a). Clinical observation on the efficacy of xiaoyaowan and guipiwan in treating sub-threshold depression of older people. W. Chin. Med. 12, 566-569. doi: 10.3969/j.issn.1673-7202.2017. 03.022

Li, S., Xu, X. Y., Zhang, L., Wu, C. Y., and Xiao, Y. L. (2017b). Treatment of 60 cases of senile subthreshold depression with Peripateticism pill combined with psychological counseling. Shanxi J. TCM 38, 769-770.

Lyness, J. M., Heo, M., Datto, C. J., Ten Have, T. R., Katz, I. R., Drayer, R., et al. (2006). Outcomes of minor and subsyndromal depression among elderly patients in primary care settings. Ann Intern Med. 144, 496-504. doi: 10.7326/ 0003-4819-144-7-200604040-00008

Mitchell, C. M., and Beals, J. (2011). The utility of the Kessler Screening Scale for Psychological Distress (K6) in two American Indian communities. Psychol. Assess. 23, 752-761. doi: 10.1037/a0023288

Morgan, A. J., Jorm, A. F., and Mackinnon, A. J. (2012). Email-based promotion of self-help for subthreshold depression: Mood Memos randomised controlled trial. Br. J. Psychiatry 200, 412-418. doi: 10.1192/bjp.bp.111.101394

Mouri, A., Ikeda, M., Koseki, T., Iwata, N., and Nabeshima, T. (2016). The ubiquitination of serotonin transporter in lymphoblasts derived from fluvoxamine-resistant depression patients. Neurosci. Lett. 617, 22-26. doi: 10. 1016/j.neulet.2016.01.064

Neumeister, A. (2003). Tryptophan depletion, serotonin, and depression: where do we stand? Psychopharmacol. Bull 37, 99-115.

Pail, G., Huf, W., Pjrek, E., Winkler, D., Willeit, M., Praschak-Rieder, N., et al. (2011). Bright-light therapy in the treatment of mood disorders. Neuropsychobiology 64, 152-162. doi: 10.1159/000328950

Pincus, H. A., Davis, W. W., and McQueen, L. E. (1999). 'Subthreshold' mental disorders. A review and synthesis of studies on minor depression and other 'brand names'. Br. J. Psychiatry 174, 288-296. doi: 10.1192/bjp.174.4.288

Raison, C. L., Capuron, L., and Miller, A. H. (2006). Cytokines sing the blues: inflammation and the pathogenesis of depression. Trends Immunol. 27, 24-31. doi: $10.1016 /$ j.it.2005.11.006

Rodríguez, M. R., Nuevo, R., Chatterji, S., and Ayuso-Mateos, J. L. (2012). Definitions and factors associated with subthreshold depressive conditions: a systematic review. BMC Psychiatry 12:181. doi: 10.1186/1471-244X-12181

Salanti, G., Higgins, J. P., Ades, A. E., and Ioannidis, J. P. (2008). Evaluation of networks of randomized trials. Stat. Methods Med. Res. 17, 279-301. doi: $10.1177 / 0962280207080643$

Sheehan, D. V., Lecrubier, Y., Sheehan, K. H., Amorim, P., Janavs, J., Weiller, E., et al. (1998). The Mini-International Neuropsychiatric Interview (M.I.N.I.): the development and validation of a structured diagnostic psychiatric interview for DSM-IV and ICD-10. J. Clin. Psychiatry 59(Suppl. 20), 22-57.

Shim, S., Yoon, B. H., Shin, I. S., and Bae, J. M. (2017). Network meta-analysis: application and practice using Stata. Epidemiol. Health 39, e2017047. doi: 10.41 78/epih.e2017047

Shima, S., Shikano, T., Kitamura, T., Masuda, Y., Tsukumo, T., Kanba, S., et al. (1984). Depression and ventricular enlargement. Acta Psychiatr. Scand. 70, 275-277. doi: 10.1111/j.1600-0447.1984.tb01208.x

Spek, V., Nyklícek, I., Smits, N., Cuijpers, P., Riper, H., Keyzer, J., et al. (2007). Internet-based cognitive behavioural therapy for subthreshold depression in people over 50 years old: a randomized controlled clinical trial. Psychol Med. 2007 37, 1797-1806. doi: 10.1017/S0033291707000542

Takagaki, K., Okamoto, Y., Jinnin, R., Mori, A., Nishiyama, Y., Yamamura, T., et al. (2018). Enduring effects of a 5-week behavioral activation program for subthreshold depression among late adolescents: an exploratory randomized controlled trial. Neuropsychiatr. Dis. Treat. 2018, 2633-2641. doi: 10.2147/NDT. S172385

Tan, M. X. (2020). Clinical study on the treatment of subthreshold depression by "xuanyang tiaoqi method" acupuncture combined with wheat moxibustion. Dissertation, Guangxi, IL: Guangxi Univ TCM, doi: 10.27879/d.cnki.ggxzy. 2020.000225

Tan, X., Liu, X., Du, J., Wu, C. Y., and Kong, J. H. (2014). Group TCM psychological intervention research on subthreshold depression population. Chin. Med. 29, 1364-1366. doi: 10.16368/j.issn.1674-8999.2014.09.031

Van Valkenhoef, G., Lu, G., de Brock, B., Hillege, H., Ades, A. E., and Welton, N. J. (2012). Automating network meta-analysis. Res. Synth. Methods 3, 285-299. doi: $10.1002 /$ jrsm. 1054

Van't Veer-Tazelaar, P. J., Van Marwijk, H. W., Van Oppen, P., Van der Horst, H. E., Smit, F., Cuijpers, P., et al. (2011). Prevention of late-life anxiety and depression has sustained effects over 24 months: a pragmatic randomized trial. Am. J. Geriatr. Psychiatry 19, 230-239. doi: 10.1097/jgp.0b013e3181faee4d

Walther, A., Lacker, T. J., and Ehlert, U. (2018). Everybody was Kung-Fu fightingThe beneficial effects of Tai Chi Qigong and self-defense Kung-Fu training on psychological and endocrine health in middle aged and older men. Complement Ther. Med. 2018, 68-72. doi: 10.1016/j.ctim.2017.11.021

Wang, Z., Ma, X., and Xiao, C. (2019). Standardized Treatment Strategy for Depressive Disorder. Adv. Exp. Med. Biol. 2019, 193-199. doi: 10.1007/978981-32-9271-0_10

Wong, S., Sun, Y. Y., Chan, A., Leung, M., Chao, D., Li, C., et al. (2018). Treating Subthreshold Depression in Primary Care: A Randomized Controlled Trial of Behavioral Activation With Mindfulness. Ann. Fam. Med. 16, 111-119. doi: 10.1370/afm.2206

Woo, J. A., Nam, Y. J., Park, Y. J., and Kwon, Y. K. (2015). Review of recent clinical trials for depression in traditional Chinese medicine Based on randomized controlled trials and systematic reviews. J. Physiol. Pathol. Korean. Med. 29, 458-466. doi: 10.15188/kjopp.2015.12.29.6.458

Xie, M. (2018). Clinical study of Yishen Tiaoqi acupuncture in the treatment of subthreshold depression of qi depression constitution. Dissertation, Beijing, IL: Beijing Univ TCM.

Xie, X. T. (2020). The effect of Tai Chi training on the depression level of young adults with subthreshold depression and its correlation with HPA axis. Dissertation, Fujian, IL: Fujian Univ TCM. dissertation.

Yin, G., Lam, C. K., and Shi, H. (2017). Bayesian randomized clinical trials: From fixed to adaptive design. Contemp. Clin. Trials. 2017, 77-86. doi: 10.1016/j.cct. 2017.04.010

Yue, N., Li, B., Yang, L., Han, Q. Q., Huang, H. J., Wang, Y. L., et al. (2018). ElectroAcupuncture Alleviates Chronic Unpredictable Stress-Induced Depressiveand Anxiety-Like Behavior and Hippocampal Neuroinflammation in Rat Model of Depression. Front. Mol. Neurosci. 11:149. doi: 10.3389/fnmol.2018. 00149

Zhang, J. Y., Ji, X. Z., Meng, L. N., and Cai, Y. J. (2019). Effects of modified mindfulness-based stress reduction (MBSR) on the psychological health of adolescents with subthreshold depression: a randomized controlled trial. Neuropsychiatr. Dis. Treat. 2019, 2695-2704. doi: 10.2147/NDT.S216401

Zhang, W. Y. (2015). Early acupuncture intervention and fMRI research on depressive syndromes of undergraduates. dissertation, Beijing, IL: Beijing Univ TCM.

Conflict of Interest: The authors declare that the research was conducted in the absence of any commercial or financial relationships that could be construed as a potential conflict of interest.

Publisher's Note: All claims expressed in this article are solely those of the authors and do not necessarily represent those of their affiliated organizations, or those of the publisher, the editors and the reviewers. Any product that may be evaluated in this article, or claim that may be made by its manufacturer, is not guaranteed or endorsed by the publisher.

Copyright (c) 2021 Jiang, Luo, Chen, Yan, Xia, Yao, Wang, He, Wang, Wang and Chen. This is an open-access article distributed under the terms of the Creative Commons Attribution License (CC BY). The use, distribution or reproduction in other forums is permitted, provided the original author(s) and the copyright owner(s) are credited and that the original publication in this journal is cited, in accordance with accepted academic practice. No use, distribution or reproduction is permitted which does not comply with these terms. 